\title{
ANALISIS DE FORMAS EN ESPECIES CHILENAS DEL GENERO TRICHOMYCTERUS (OSTEICHTHYES: SILURIFORMES) UTILIZANDO MORFOMETRIA GEOMETRICA
}

\author{
SHAPE ANALYSIS IN CHILEAN SPECIES OF TRICHOMYCTERUS \\ (OSTEICHTHYES: SILURIFORMES) USING GEOMETRIC MORPHOMETRY
}

\author{
R. Pardo, S. Scott \& I. Vila \\ Laboratorio de Limnología, Departamento de Ciencias Ecológicas, Universidad de Chile \\ Email: rodrigo@abulafia.ciencias.uchile.cl
}

\begin{abstract}
RESUMEN
El género Trichomycterus incluye aproximadamente 120 especies, que se distribuyen comúnmente en las cabeceras de los ríos neotropicales. En Chile este género se encuentra ampliamente distribuido: en el altiplano $\left(14^{\circ}-22^{\circ} \mathrm{S}\right)$ Trichomycterus chungaraensis; T. laucaensis y T. rivulatus, mientras T. areolatus se distribuye entre Huasco $\left(28^{\circ} 27^{\prime} \mathrm{S}\right)$ y Chiloé $\left(41^{\circ} 27^{\prime} \mathrm{S}\right)$, presentando la mayor amplitud en extensión, y en simpatría con $T$. chiltoni en la cuenca del Bío Bío ( $36^{\circ} 49^{\prime}$ S). La diversidad de hábitats colonizados por este género habría conllevado cambios morfológicos, los que se analizaron con la técnica morfometría geométrica. Se localizaron siete landmarks del tipo I y II, y se efectuó una superposición de landmarks por el método de GPA (Generalized Procrustes Superimposition Analysis). Para describir las relaciones entre las especies se utilizó un análisis de Relative Warp, en el cual los dos primeros Relative Warp explicaron el 67,22\%. Con una ordenación de tipo UPGMA se encontró una fuerte relación entre $T$. chungaraensis, $T$. laucaensis y $T$. rivulatus, diferenciándose de $T$. areolatus y $T$. chiltoni, los que mostraron alta similitud entre ellos. Estas relaciones de forma se asociarían con las distribuciones geográficas de las especies, correspondientes a la provincia del Titicaca y a la provincia Chilena, respectivamente.
\end{abstract}

Palabras Claves: Provincias ictiogeográficas, landmarks, Relative Warp, Chile.

\section{ABSTRACT}

The genus Trichomycterus includes nearly 120 species that generally inhabits at the neotropical river headwaters. In Chile this genus has a wide distribution: T. chungaraensis, T. laucaensis and T. rivulatus live at the altiplano, while $T$. areolatus distributes from the III to the $\mathrm{X}$ chilean regions with the wider extension living in simpatry with T. chiltoni in Biobio basin. The habitat diversities colonized by this genus would have driven morphological changes that were analyzed by geometric morphology. Seven type I and II landmarks were fixed, and a superposition was done by GPA (General Procruster Analysis). To describe relations between species RWA (Relative warps Analysis) was used, in which the first two RW explain 67, 22\% and a UPGMA was performed. A strong relationship between $T$. chungaraensis, T. laucaensis and T. rivulatus was found; being different from T. areolatus and $T$. chiltoni which were similar. These form relationship would be associated with geographic species distributions that correspond to Titicaca and Chilean provinces respectively.

KEYwords: Ichthyogeographic Provinces, landmarks, Relative Warp, Chile. 


\section{INTRODUCCION}

Trychomicteridae forma parte de las 32 familias pertenecientes al orden Siluriformes, uno de los grupos de peces teleósteos más diversos en todo el mundo. Este grupo aparentemente presenta una falta de sinapomorfías (Arratia 1990; de Pinna 1998), que hacen difícil la clasificación o relación entre especies. Trichomycterinae es la única subfamilia representada en las regiones brasileña y austral (Arratia 1990). En los sistemas acuáticos chilenos de la provincia del Titicaca (Ringuelet 1975), en la región biogeográfica andina, se encuentra Trichomycterus rivulatus Valenciennes 1848 que es la especie con mayor distribución abarcando sistemas chilenos, bolivianos y peruanos; Trichomycterus laucaensis Arratia, 1983 se encuentra asociado a la cuenca del río Lauca, y Trichomycterus chungaraensis Arratia, 1983 está circunscrito a una de las vertientes que alimentan el lago Chungará, todos estos sistemas se encuentran sobre los $4.000 \mathrm{msnm}$. En la zona austral (Ringuelet 1975), en la provincia Chilena, se encuentra Trichomycterus areolatus Valenciennes, 1848, especie predominante, que se distribuye desde Huasco $\left(28^{\circ} 27^{\prime} \mathrm{S}\right)$ hasta la isla grande de Chiloé $\left(41^{\circ} 27^{\prime}\right.$ 'S) y Trichomycterus chiltoni (Eigenmann, 1928), distribuido desde Concepción (36²9'S) a Osorno (40³4'S), estas dos últimas especies se encuentran en simpatría en la cuenca del río Biobío. El desconocimiento de las relaciones filogenéticas existentes entre estas especies, tomando en cuenta su amplia distribución, así como su adaptación a las distintas zonas y tratándose en su mayoría de especies en peligro de extinción, hacen necesario e importante conocer las relaciones entre ellos. El objetivo de este trabajo es encontrar relaciones de forma entre las especies chilenas de Trichomycterus, aunque este ordenamiento no considera las relaciones históricas entre las especies, debido a que los puntos homólogos utilizados no se pueden considerar como homologías verdaderas (necesarias para realizar filogenias), pero sí podrían dar luces de las interacciones que estas especies han tenido con su ambiente desde su separación.

\section{MATERIALES Y METODOS}

Se analizó un total de 39 individuos de las cinco especies de Trichomycterus descritas para Chile, analizándose cinco ejemplares de la especie Trichomycterus areolatus provenientes de la cuenca del río Choapa (31 $39^{\circ} \mathrm{S} ; 71^{\circ} 18^{\prime} \mathrm{W}$ ), ocho de $T$. chiltoni del río Pangue, de la cuenca del Bío Bío ( $\left.37^{\circ} 55^{\prime} \mathrm{S} ; 71^{\circ} 36^{\prime} \mathrm{W}\right)$, nueve de $T$. rivulatus provenientes del río Isluga ( $\left(1^{\circ} 15^{\prime} \mathrm{S} ; 68^{\circ} 40^{\prime} \mathrm{W}\right)$, ocho de T. laucaensis del río Lauca $\left(18^{\circ} 11^{\prime} \mathrm{S} ; 69^{\circ} 20^{\prime} \mathrm{W}\right)$ y nueve ejemplares de T. chungaraensis de la vertiente Mal Paso $\left(18^{\circ} 16^{\prime} \mathrm{S} ; 69^{\circ} 10^{\prime} \mathrm{W}\right)$ en la reserva Nacional Lauca.

Los individuos fueron fotografiados en vista lateral con una cámara digital de alta resolución; en cada una de las fotografías se identificaron siete landmarks o puntos anatómicamente homólogos (Fig. 1). Las coordenadas de los especímenes fueron alineadas (trasladadas, rotadas y escaladas ajustándose unas a otras) usando el método generalizado de superimposición de procrustes, por mínimos cuadrados (con sigla en inglés GLS) este método ajusta una configuración sobre otra minimizando la suma de los cuadrados de las distancias entre landmarks homólogos (Rohlf 1990, Rohlf \& Slice 1990). Mediante este procedimiento se eliminó la información de tamaño y posición de las coordenadas. Luego se obtuvieron los resultados de Relative Warp, que son los componentes principales de la variación entre especímenes en el espacio multivariado; estos valores constituyen la serie de datos multivariados de forma, con los que se realizó el análisis de ordenación por distancias euclidianas, utilizando el algoritmo de UPGMA (Sneath \& Snokal 1973).

\section{RESULTADOS}

Los primeros dos ejes del análisis de Relative Warp explican el $49,87 \%$ y $17,35 \%$, respectivamente, de la varianza de los datos de forma. Las distancias euclidianas, calculadas utilizando los datos de Relative Warp, entre especies de Trichomycterus indican una mayor relación entre las especies que habitan una misma zona biogeográfica (Fig. 2), agrupándose en un mismo cluster $T$. chungarensis, T. laucaensis y $T$. rivulatus, que corresponden a especies de la provincia del Titicaca. Mientras T. areolatus y $T$. chiltoni forman un grupo aparte, habitando en la provincia Chilena. 


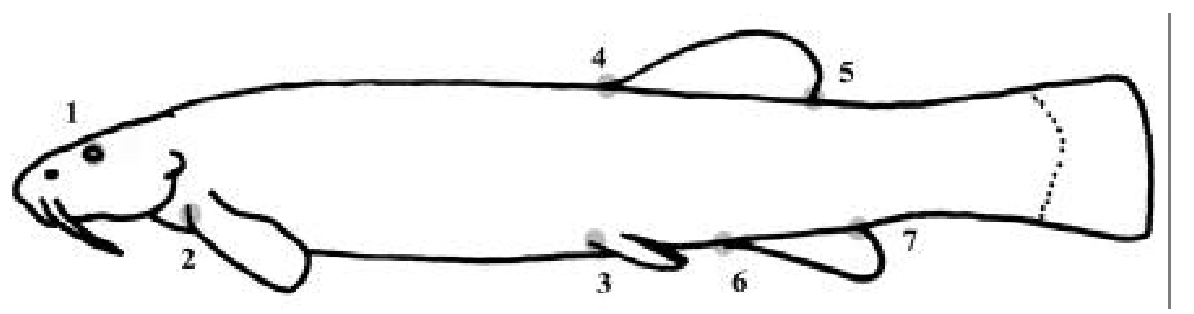

FiguRa 1. Landmarks utilizados: (1) centrointerior del ojo, (2) inserción de la aleta pectoral, (3) inserción de la aleta pélvica, (4) inicio y (5) término de la aleta dorsal, (6) inserción y (7) término de la aleta anal.

FIGURE 1. Utilized landmarks: (1) eye center, (2) pectoral fin insertion, (3) pelvic fin inserion, (4) beginning and (5) end of dorsal fin, (6) beginning and (7) end of anal fin.

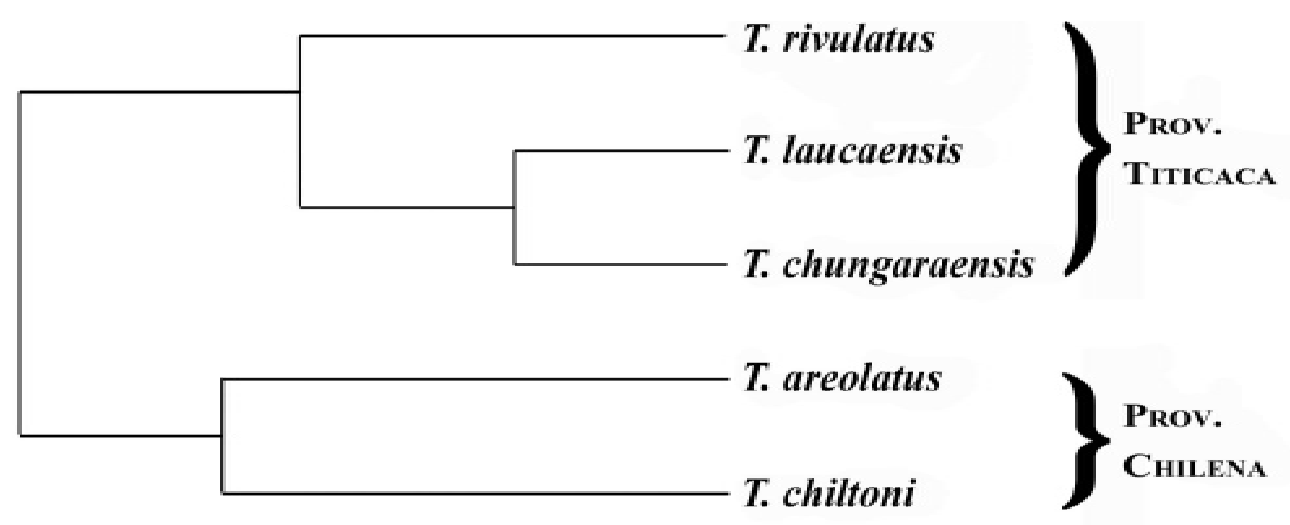

\begin{tabular}{|c|c|c|c|c|c|}
\hline 0,12 & 0,1 & 0,08 & 0,06 & 0,04 & 0,02 \\
\hline
\end{tabular}

FIGURA 2. Fenograma UPGMA construido con las distancias Euclidianas computadas con todos los partial warps.

FIGURA 2. UPGMA phenogram constructed from Euclidean distances computed on all partial warps.

\section{DISCUSION}

Basado en la distribución de especies ícticas Ringuelet (1975) describió las zonas ictiográficas de América del Sur. Asociado con esta descripción él asignó a la fauna sudamericana un fuerte endemismo (Ringuelet et al.1967), el territorio chileno se inserta en las provincias ictiográficas de Titicaca, Chilena y Patagónica (Ringuelet 1975; Arratia 1997; Dyer 2000).

Trichomycterus, a pesar de ser el grupo más diverso en las provincias ictiograficas continentales de Chile, presenta falencias en el conocimiento de su sistemática, ecología y biología (Dyer 2000; Vila et al. 1999). Las relaciones entre especies de Trichomycterus están lejos de estar elucidadas, siendo éste uno de los desafíos pendientes en la sistemática de Siluriformes Neotropicales (De Pinna 1998). Este trabajo señala que las especies de Trichomycterus, que habitan el territorio chileno, se agrupan, según sus formas, en dos cluster principales, correspondientes a las provincias del Titicaca y Chilena. En la provincia del Titicaca los ríos son de alturas superiores a los $4.000 \mathrm{msnm}$, se caracterizan 
por ser cortos y de bajo caudal (Niemeyer \& Cereceda 1984). A su vez los ríos de la provincia Chilena presentan caudales mayores, más extensos (Niemeyer \& Cereceda 1984). Estas características ambientales podrían estar modificando la forma de los peces que habitan estas provincias, diferenciándolas entre ellas, lo que se acrecentaría por la presencia de una extensa área sin peces que separa estas dos zonas (Ringuelet 1975).

\section{BIBLIOGRAFIA}

Arratia, G. 1990. The South American Trichomycterinae (Teleostei: Siluriformes), a problematic group. Pp: 395-403. In: G. Peters \& R. Hutterer (eds.), Vertebrates in the Tropics. Museum Alexander Koening, Bonn.

Arratia, G. 1997. Brazilian and Austral freshwater fish faunas of South America. A contrast. H. Ulrich (ed.): Tropical biodiversity and systematics. Procedings of the international Symposium on Biodiversity and Systematics in Tropical Ecosistems, Museum Alexander Koenig, Bonn.

De Pinna, M.C. 1998. Phylogenetic relationship of Neotropical siluriforms (Teleostei: Ostariophysi): historical overview and synthesis of hypotheses. En Phylogeny and classification of Neotropical fishes. Malabarba, L.R., R.E. Reis, R.P. Vari, Z.M. Lucena \& C.A. Lucena (eds.). Porto Alegre, Edipucrs, pp: 279-330.

DYer, B. 2000. Systematic review and biogeography of the freshwater fishes of Chile. Estudios Oceanológicos (Chile). 19: 77-98.

Niemeyer, H. \& P. Cereceda. 1984. Hidrografía. Geografía de Chile. Tomo VIII. Instituto Geográfico Militar. Chile.

RiNGUELET, R.A. 1975. Zoogeografía y ecología de los peces de aguas continentales de la Argentina y consideraciones sobre las áreas ictiológicas de América del Sur. Ecosur, Corrientes. 2: 1-151.

Ringuelet, R.A., R.H. Aramburu, D.E. Aramburu \& A. Alonso. 1967. Los peces argentinos de agua dulce. Gobernación de la provincia de Buenos Aires. Comisión de Investigación Científica, La Plata.

RoHLF, F.J. 1990. Rotacional fit (Procrustes) methods. In: F.J. Rohlf \& F.L. Bookstein, eds. Proceedings of the Michigan Morphometrics Workshop. Special Publication $\mathrm{N}^{\circ}$ 2. An Arbor: Univ. of Michigan Museum of Zoology. Pp: 227-236.

Rohlf, F.J. \& D.E. Slice. 1990. Extensions of the Procrustes method for the optimal superimposition of landmarks. Syst. Zool. 39: 40-59.

Sneath, P.H. \& R.R. Sokal. 1973. Numerical Taxonomy San Francisco: Freeman.

Vila, I., L. Fuentes \& M. Contreras. 1999. Peces límnicos de Chile. Boletín del Museo Nacional de Historia Natural, Chile. 48: 61-75. 